Volume 359, Number 12, December 2007, Pages 5899-5913

S 0002-9947(07)04238-9

Article electronically published on June 26, 2007

\title{
QUASILINEAR ELLIPTIC EQUATIONS WITH BMO COEFFICIENTS IN LIPSCHITZ DOMAINS
}

\author{
SUN-SIG BYUN AND LIHE WANG
}

\begin{abstract}
We obtain a global $W^{1, q}$ estimate for the weak solution to an elliptic partial differential equation of $p$-Laplacian type with BMO coefficients in a Lipschitz domain with small Lipschitz constant.
\end{abstract}

\section{INTRODUCTION}

Suppose that $1<p<\infty$. We are concerned with the following equation:

$$
\operatorname{div}\left((A \nabla u \cdot \nabla u)^{\frac{p-2}{2}} A \nabla u\right)=\operatorname{div}\left(|\mathbf{f}|^{p-2} \mathbf{f}\right) \text { in } \Omega,
$$

where $\Omega$ is an open, bounded subset of $\mathbb{R}^{n}$. The coefficients matrix $A$ is assumed to be essentially bounded and uniformly elliptic; that is,

$$
A \in L^{\infty}(\Omega)
$$

and

$$
\Lambda^{-1}|\xi|^{2} \leq A(x) \xi \cdot \xi \leq \Lambda|\xi|^{2}
$$

for some $\Lambda>0$, a.e. $x \in \mathbb{R}^{n}$ and all $\xi \in \mathbb{R}^{n}$. We assume as well

$$
\mathbf{f} \in L^{q}(\Omega)
$$

for some $q \geq p$.

We are interested in the question: What is a minimal requirement on the coefficients matrix $A$ and a more general geometric condition on the boundary of $\Omega$ on which $W^{1, q}$ estimates hold? In particular we are interested in estimates like

$$
\int_{\Omega}|\nabla u|^{q} d x \leq C \int_{\Omega}|\mathbf{f}|^{q} d x
$$

for some constant $C$ independent of $u$ and $\mathbf{f}$.

This is a classical question, and there have been many works in this direction (see e.g. $[4,6,10,11])$. In $[10,11]$ the authors considered the Dirichlet problem for (1.1) to prove the well posedness in $W^{1, q}(\Omega)$ under the assumptions that $A$ is

Received by the editors August 5, 2005.

2000 Mathematics Subject Classification. Primary 35R05, 35R35; Secondary 35J15, 35J25.

Key words and phrases. $W^{1, p}$ estimates, quasilinear elliptic equations, BMO space, Lipschitz domain, maximal function, Vitali covering lemma.

The first author was supported in part by KRF-2005-003-C00016.

The second author was supported in part by NSF Grant \#0401261. 
of the space VMO and that $\partial \Omega$ is locally $C^{1, \alpha}, 0<\alpha \leq 1$. There the authors used the sharp maximal functions and found a local version for the the sharp maximal functions while here we will use maximum functions and simplify their proof.

Our work is very much influenced by $[3,14]$ and the works in $[10,11]$. We are working under the assumption that the boundary $\partial \Omega$ of the domain is locally, the graph of a function which is required to be Lipschitz continuous (see papers $[7,8,9])$.

Recently in [1] the author dealt with PDE (1.1) when $p=2$ with zero boundary condition to show that $W^{1, q}$ estimates hold under the assumptions that $A$ has small BMO seminorms and that $\Omega$ has locally, small Lipschitz constants. The author used a scaling based on the standard $L^{p}$ estimates, maximal functions and a Vitali covering lemma. The key idea is to find the decay estimates of the Hardy-Littlewood maximal function of the gradient of solutions. This approach used in $[1,14]$ enables the authors to avoid the classical one which uses integral representations.

In this work zero boundary condition is studied. More precisely, we consider the following Dirichlet problem:

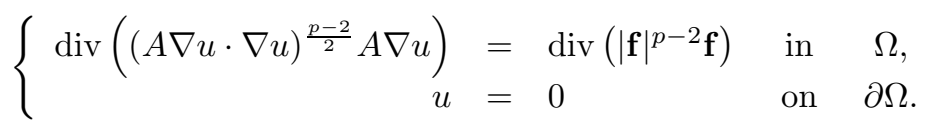

Definition 1.1. Weak solution of (1.3) is a function $u \in W_{0}^{1, q}(\Omega)$ such that

$$
\int_{\Omega}(A \nabla u \cdot \nabla u)^{\frac{p-2}{2}} A \nabla u \cdot \nabla \varphi d x=\int_{\Omega}|\mathbf{f}|^{p-2} \mathbf{f} \cdot \nabla \varphi d x
$$

for all $\varphi \in C_{0}^{\infty}(\Omega)$.

We refer to $[10,11]$ for a general discussion on equation (1.1).

The main theorem is stated as follows.

Theorem 1.2. Let $q>p$. Then there is a small $\delta=\delta(\Lambda, p, n, R)>0$ so that for all $A$ with $A(\delta, R)$-vanishing, for all $\Omega$ with $\Omega(\delta, R)$-vanishing, and for all $\mathbf{f}$ with $\mathbf{f} \in L^{q}\left(\Omega ; \mathbb{R}^{n}\right)$, the Dirichlet problem (1.3) has a unique weak solution with the estimate

$$
\int_{\Omega}|\nabla u|^{q} d x \leq C \int_{\Omega}|\mathbf{f}|^{q} d x
$$

where the constant $C$ is independent of $u$ and $\mathbf{f}$.

We wish to conclude this Introduction by mentioning that a Lipschitz domain with small Lipschitz constant exhibits the minimal geometric condition necessary for the $W^{1, q}$ regularity theory in this direction, from the point of view that the boundary of the domain is locally the graph of a function.

\section{Preliminaries}

In this section we describe precisely the assumptions considered in this work on the coefficients matrix $A$ and the boundary $\partial \Omega$ of the domain $\Omega$. Here we also introduce the main tools we will use. 
In view of [12], $A$ is assumed to be defined on $\mathbb{R}^{n}$. For $x \in \mathbb{R}^{n}$ and $r>0$, $B_{r}(x)$ denotes an $n$-dimensional ball of radius $r$ and center $x$. We use the following definition.

Definition 2.1. We say that the coefficients matrix $A$ is $(\delta, R)$-vanishing if

$$
\sup _{0<r \leq R} \sup _{x \in \mathbb{R}^{n}} \frac{1}{\left|B_{r}\right|} \int_{B_{r}(x)}\left|A(y)-\bar{A}_{B_{r}(x)}\right|^{\frac{p}{p-1}} d y \leq \delta^{\frac{p}{p-1}} .
$$

$\partial \Omega$ is assumed to be written locally as the graph of Lipschitz functions with small Lipschitz norms.

Definition 2.2. We say that $\Omega$ is $(\delta, R)$-Lipschitz if for every $x_{0} \in \partial \Omega$ and every $r \in(0, R]$, there exists a Lipschitz continuous function $\gamma: \mathbb{R}^{n-1} \rightarrow \mathbb{R}$ with $\operatorname{Lip}(\gamma) \leq$ $\delta$ such that

$$
\Omega \cap B_{r}\left(x_{0}\right)=\left\{x=\left(x_{1}, \ldots, x_{n-1}, x_{n}\right)=\left(x^{\prime}, x_{n}\right) \in B_{r}\left(x_{0}\right): x_{n}>\gamma\left(x^{\prime}\right)\right\}
$$

in some coordinate system.

We remark that one might assume that $R$ in the definitions above to be 1 by scaling the given equations, while $\delta$ is scaling invariant. Through this paper we mean $\delta$ to be a small positive constant.

We will combine the compactness method, the classical Hardy-Littlewood maximal function, the Vitali covering lemma and standard arguments of measure theory.

Our compactness method is based on the following lemma:

Lemma 2.3 ([12]). If $\Omega$ is a Lipschitz domain, then $W^{1, p}(\Omega)$ is compactly embedded in $L^{p}(\Omega)$ for all $1<p<\infty$.

We use a maximal function argument.

Definition 2.4. The Hardy-Littlewood maximal function $\mathcal{M} f$ of a locally integrable function $f$ is a function such that

$$
(\mathcal{M} f)(x)=\sup _{r>0} \frac{1}{\left|B_{r}(x)\right|} \int_{B_{r}(x)}|f(y)| d y,
$$

where $B_{r}(x)$ is the open ball of radius $r$ centered at $x$.

$$
\mathcal{M}_{\Omega} f=\mathcal{M}\left(f \chi_{\Omega}\right),
$$

if $f$ is not defined outside $\Omega$. ing.

The basic properties for the Hardy-Littlewood maximal function are the follow-

Lemma 2.5 ([13]). (1) (strong $p$-p estimate). If $f \in L^{p}\left(\mathbb{R}^{n}\right)$ with $1<p \leq \infty$, then $\mathcal{M} f \in L^{p}\left(\mathbb{R}^{n}\right)$ and

$$
\frac{1}{C}\|f\|_{L^{p}} \leq\|\mathcal{M} f\|_{L^{p}} \leq C\|f\|_{L^{p}} .
$$

(2) (weak 1-1 estimate). If $f \in L^{1}\left(\mathbb{R}^{n}\right)$, then

$$
\left.\mid\left\{x \in \mathbb{R}^{n}:(\mathcal{M} f)\right)(x)>t\right\}\left|\leq \frac{C}{t} \int\right| f(x) \mid d x .
$$


We will use the following version of the Vitali covering lemma.

Lemma 2.6 ([1]). Assume that $C$ and $D$ are measurable sets with $C \subset D \subset B_{1}^{+}$. We suppose further that there exists an $\epsilon>0$ such that

$$
|C|<\epsilon\left|B_{1}^{+}\right|
$$

and

$$
\text { for every } x \in B_{1}^{+} \text {with }\left|C \cap B_{r}(x)\right| \geq \epsilon\left|B_{r}\right|, B_{r}(x) \cap B_{1}^{+} \subset D .
$$

Then we have

$$
|C| \leq 2(10)^{n} \epsilon|D|
$$

We use the following standard arguments of measure theory.

Lemma $2.7([2])$. Suppose that $f$ is a nonnegative and measurable function in $\mathbb{R}^{n}$. Suppose further that $f$ has a compact support in a bounded subset $E$ of $\mathbb{R}^{n}$. Let $\theta>0$ and $m>1$ be constants. Then for $0<p<\infty$ we have

$$
f \in L^{p}(E) \Longleftrightarrow S=\sum_{k \geq 1} m^{k p}\left|\left\{x \in E: f(x)>\theta m^{k}\right\}\right|<\infty
$$

and

$$
\frac{1}{C} S \leq\|f\|_{L^{p}(E)}^{p} \leq C(|E|+S)
$$

where $C>0$ is a constant depending only on $\theta, m$, and $p$.

\section{INTERIOR REGULARITY}

With the different types of equations from those in [1] we should start out with the definition of weak solutions concerning (1.1). Based on a scaling we consider the following PDE:

$$
\operatorname{div}\left((A \nabla u \cdot \nabla u)^{\frac{p-2}{2}} A \nabla u\right)=\operatorname{div}\left(|\mathbf{f}|^{p-2} \mathbf{f}\right) \text { in } B_{6} .
$$

Definition 3.1. A weak solution of (3.1) is a function $u \in W^{1, p}\left(B_{6}\right)$ which satisfies

$$
\int_{B_{6}}(A \nabla u \cdot \nabla u)^{\frac{p-2}{2}} A \nabla u \cdot \nabla \varphi d x=\int_{B_{6}}|\mathbf{f}|^{p-2} \mathbf{f} \cdot \nabla \varphi d x
$$

for all $\varphi \in C_{0}^{\infty}\left(B_{6}\right)$.

One can prove the following interior $W^{1, q}$ estimates via the same lines of ideas considered in $W^{1, q}$ boundary estimates (see Section 4 ).

Theorem 3.2. Let $q$ be a real number with $q>p$. Then there is a small $\delta=$ $\delta(\Lambda, p, n)>0$ so that for all $A$ with $A(\delta, 6)$-vanishing, if $u$ is a weak solution of (3.1), then $u$ belongs to $W^{1, q}\left(B_{1}\right)$ with the estimate

$$
\|\nabla u\|_{L^{q}\left(B_{1}\right)} \leq C\left(\|u\|_{L^{q}\left(B_{6}\right)}+\|\mathbf{f}\|_{L^{q}\left(B_{6}\right)}\right),
$$

where the constant $C$ is independent of $u$ and $\mathbf{f}$. 
The main thing to do for the proof of the theorem above is to derive the following lemma. One can find its proof in the same way as we will treat Lemma 4.5.

Lemma 3.3. There is a constant $N_{1}>0$ so that for any $0<\epsilon, r \leq 1$, there exists a small $\delta=\delta(\epsilon)>0$ such that if $u$ is a weak solution of (3.1), with $A$ $(\delta, 6)$-vanishing and

$$
\left|\left\{x \in B_{1}(0): \mathcal{M}\left(|\nabla u|^{p}\right)(x)>N_{1}^{p}\right\} \cap B_{r}\right| \geq \epsilon\left|B_{r}\right|,
$$

then we have

$$
B_{r} \cap B_{1}(0) \subset\left\{x \in B_{1}(0): \mathcal{M}\left(|\nabla u|^{p}\right)>1\right\} \cup\left\{x \in B_{1}(0): \mathcal{M}\left(|\mathbf{f}|^{p}\right)>\delta^{p}\right\},
$$

where $B_{r}$ denotes the ball with radius $r$ and center in $B_{1}(0)$.

\section{Boundary REgularity}

Now we are intended to find the boundary $W^{1, q}$ regularity with $p<q<\infty$ regarding the Dirichlet problem (1.3) under the assumptions that the coefficients matrix is $(\delta, R)$-vanishing and the domain is $(\delta, R)$-Lipschitz. The important analytical tools are the maximal function and a modified Vitali covering lemma (see Section 2 of paper [1]). As the boundary of the domain is, locally, the graph of a function which is Lipschitz continuous, we are first concerned with boundary estimates on flat boundaries.

Denote

$$
\begin{gathered}
T_{R}=B_{R} \cap\left\{x_{n}=0\right\}, T_{R}\left(x_{0}\right)=T_{R}+x_{0}^{\prime} \text { for } x_{0}^{\prime} \in \mathbb{R}^{n-1}, \\
B_{R}^{+}=B_{R} \cap\left\{x_{n}>0\right\}, B_{R}^{+}\left(x_{0}\right)=B_{R}\left(x_{0}\right) \cap\left\{x_{n}>0\right\} \text { for } x_{0} \in \mathbb{R}^{n} .
\end{gathered}
$$

Definition 4.1. We say that $u \in W^{1, q}\left(B_{R}^{+}\right)$is a weak solution of

$$
\left\{\begin{aligned}
\operatorname{div}\left((A \nabla u \cdot \nabla u)^{\frac{p-2}{2}} A \nabla u\right) & =\operatorname{div}\left(|\mathbf{f}|^{p-2} \mathbf{f}\right) & & \text { in } B_{R}^{+}, \\
u & =0 & & \text { on } T_{R},
\end{aligned}\right.
$$

if we have

$$
\int_{B_{R}^{+}}(A \nabla u \cdot \nabla u)^{\frac{p-2}{2}} A \nabla u \cdot \nabla \varphi d x=\int_{B_{R}^{+}}|\mathbf{f}|^{p-2} \mathbf{f} \cdot \nabla \varphi d x
$$

for all $\varphi \in C_{0}^{\infty}\left(B_{R}^{+}\right)$and the zero extension of $u$ is of $W^{1, p}\left(B_{R}\right)$.

The compactness argument is based on the following observation: Since $A$ is $(\delta, R)$-vanishing, one can freeze the constant coefficients $\bar{A}_{B_{R}^{+}}$and use known integral estimates of the reference equation of (4.1)

$$
\left\{\begin{array}{rllll}
\operatorname{div}\left(\left(\bar{A}_{B_{R}^{+}} \nabla v \cdot \nabla v\right)^{\frac{p-2}{2}} \bar{A}_{B_{R}^{+}} \nabla v\right) & =0 & \text { in } & B_{R}^{+}, \\
v & = & 0 & \text { on } & T_{R}
\end{array}\right.
$$

to observe that $u-v$ is small in $L^{P}\left(B_{R}^{+}\right)$provided that $\mathbf{f}$ is small in $L^{P}\left(B_{R}^{+}\right)$, which is possible since it is about data, and that $A$ is small in $B M O$, which is the assumption imposed on $A$.

Lemma 4.2. For any $\epsilon>0$, there exists a small $\delta=\delta(\epsilon)>0$ such that if $u$ is a weak solution of (4.1) in $B_{4}^{+}$, with

$$
\frac{1}{\left|B_{4}\right|} \int_{B_{4}^{+}}\left|A-\bar{A}_{B_{4}^{+}}\right|^{\frac{p}{p-1}} d x \leq \delta^{\frac{p}{p-1}}
$$


and

$$
\frac{1}{\left|B_{4}\right|} \int_{B_{4}^{+}}|\nabla u|^{p} d x \leq 1, \frac{1}{\left|B_{4}\right|} \int_{B_{4}^{+}}|\mathbf{f}|^{p} d x \leq \delta^{p},
$$

then there exist a weak solution $v$ of (4.2) in $B_{4}^{+}$such that

$$
\int_{B_{4}^{+}}|u-v|^{p} d x \leq \epsilon^{p} .
$$

Proof. We argue by contradiction. If not, there would exist $\epsilon_{0}>0,\left\{A_{k}\right\}_{k=1}^{\infty}$, $\left\{u_{k}\right\}_{k=1}^{\infty}$ and $\left\{\mathbf{f}_{k}\right\}_{k=1}^{\infty}$ such that $u_{k}$ is a weak solution of

$$
\left\{\begin{aligned}
\operatorname{div}\left(\left(A_{k} \nabla u_{k} \cdot \nabla u_{k}\right)^{\frac{p-2}{2}} A_{k} \nabla u_{k}\right) & =\operatorname{div}\left(\left|\mathbf{f}_{\mathbf{k}}\right|^{p-2} \mathbf{f}_{\mathbf{k}}\right) & \text { in } B_{4}^{+}, \\
u_{k} & =0 & \text { on } T_{4},
\end{aligned}\right.
$$

with

$$
\frac{1}{\left|B_{4}\right|} \int_{B_{4}^{+}}\left|A_{k}-{\overline{A_{k}}}_{B_{4}^{+}}\right|^{\frac{p}{p-1}} d x \leq \frac{1}{k^{\frac{p}{p-1}}}
$$

and

$$
\frac{1}{\left|B_{4}\right|} \int_{B_{4}^{+}}\left|\nabla u_{k}\right|^{p} d x \leq 1, \frac{1}{\left|B_{4}\right|} \int_{B_{4}^{+}}\left|\mathbf{f}_{k}\right|^{p} d x \leq \frac{1}{k^{p}} .
$$

But we have

$$
\int_{B_{4}^{+}}\left|u_{k}-v_{k}\right|^{p} d x>\epsilon_{0}^{p}
$$

for any weak solution $v_{k}$ of

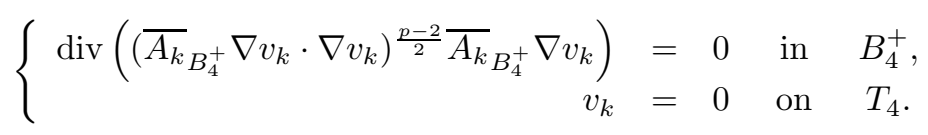

Noting that $u_{k}=0$ on $T_{4}$ and using (4.8), we observe that $\left\{u_{k}\right\}_{k=1}^{\infty}$ is bounded in $W^{1, p}\left(B_{4}^{+}\right)$. Consequently there exists a subsequence, which we still denote by $\left\{u_{k}\right\}$, and $u_{0} \in W^{1, p}\left(B_{4}^{+}\right)$such that

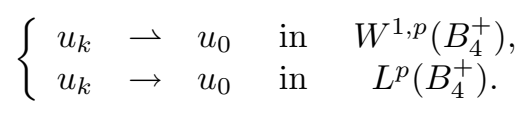

As $\left\{\bar{A}_{k_{3}}\right\}_{k=1}^{\infty}$ is bounded in $l^{\infty}$, there exists a subsequence, which we denote by $\left\{\overline{A_{k}}\right\}$, such that

$$
\overline{A_{k}} \rightarrow A_{0} \text { in } l^{\infty}
$$

for some constant coefficients matrix $A_{0}$. Consequently (4.7) implies

$$
A_{k} \rightarrow A_{0} \text { in } L^{\frac{p}{p-1}}\left(B_{4}^{+}\right) .
$$

Now we want verify that $u_{0}$ is a weak solution of

$$
\left\{\begin{array}{rllll}
\operatorname{div}\left(\left(A_{0} \nabla u_{0} \cdot \nabla u_{0}\right)^{\frac{p-2}{2}} A_{0} \nabla u_{0}\right) & = & 0 & \text { in } & B_{4}^{+}, \\
u_{0} & = & 0 & \text { on } & T_{4} .
\end{array}\right.
$$

Fix any $\varphi \in C_{0}^{\infty}\left(B_{4}^{+}\right)$. Then we recall Definition 4.1 to find from (4.6) that

$$
\int_{B_{4}^{+}}\left(A_{k} \nabla u_{k} \cdot \nabla u_{k}\right)^{\frac{p-2}{2}} A_{k} \nabla u_{k} \cdot \nabla \varphi d x=\int_{B_{4}^{+}}\left|\mathbf{f}_{k}\right|^{p-2} \mathbf{f}_{k} \cdot \nabla \varphi d x .
$$


Now using (4.12), (4.11), the method of Minty (see Theorem 3 of Chapter 9 in [5]), and (4.8) and letting $k \rightarrow \infty$ in (4.14), we have

$$
\int_{B_{4}^{+}}\left(A_{0} \nabla u_{0} \cdot \nabla u_{0}\right)^{\frac{p-2}{2}} A_{0} \nabla u_{0} \cdot \nabla \varphi d x=0 .
$$

This establishes (4.13) since $u_{0}=0$ in $T_{4}$ in the trace sense from (4.11). Taking $v=u_{0}$ and sending $k \rightarrow \infty$, we reach a contradiction to (4.10).

Lemma 4.3. There is a constant $N_{1}>0$ so that for any $\epsilon>0$, there exists a small $\delta=\delta(\epsilon)>0$ such that if $u$ is a weak solution of (4.1) in $B_{6}^{+}$, with

$$
\frac{1}{\left|B_{4}\right|} \int_{B_{4}^{+}}\left|A-\bar{A}_{B_{4}^{+}}\right|^{\frac{p}{p-1}} d x \leq \delta^{\frac{p}{p-1}}
$$

and

$$
B_{1}^{+} \cap\left\{x \in B_{6}^{+}: \mathcal{M}\left(|\nabla u|^{p}\right)(x) \leq 1\right\} \cap\left\{x \in B_{6}^{+}: \mathcal{M}\left(|\mathbf{f}|^{p}\right)(x) \leq \delta^{p}\right\} \neq \emptyset,
$$

then we have

$$
\left|\left\{x \in B_{6}^{+}: \mathcal{M}\left(|\nabla u|^{p}\right)(x)>N_{1}^{p}\right\} \cap B_{1}^{+}\right|<\epsilon\left|B_{1}\right| .
$$

Proof. In view of (4.16), there exists an $x_{0} \in B_{1}^{+}$such that

$$
\frac{1}{\left|B_{r}\right|} \int_{B_{r}\left(x_{0}\right)}|\nabla u|^{p} d x \leq 1, \frac{1}{\left|B_{r}\right|} \int_{B_{r}\left(x_{0}\right)}|\mathbf{f}|^{p} d x \leq \delta^{p}
$$

for all $r>0$. Now that $B_{4}^{+}(0) \subset B_{5}^{+}\left(x_{0}\right)$ we see from (4.18) that

$$
\frac{1}{\left|B_{4}\right|} \int_{B_{4}^{+}(0)}|\mathbf{f}|^{p} d x \leq\left(\frac{5}{4}\right)^{n} \frac{1}{\left|B_{5}\right|} \int_{B_{5}^{+}\left(x_{0}\right)}|\mathbf{f}|^{p} d x \leq\left(\frac{5}{4}\right)^{n} \delta^{p}
$$

and

$$
\frac{1}{\left|B_{4}\right|} \int_{B_{4}^{+}(0)}|\nabla u|^{p} d x \leq\left(\frac{5}{4}\right)^{n}
$$

Applying the lemma above to the PDE (4.1), with $\left(\frac{4}{5}\right)^{n} u$ replacing $u$ and $\left(\frac{4}{5}\right)^{n} \mathbf{f}$ replacing $\mathbf{f}$, we deduce that for any $\eta>0$, there exist a small $\delta(\eta)$ and a corresponding weak solution $v$ of $(4.2)$ in $B_{4}^{+}$such that

$$
\int_{B_{4}^{+}}|u-v|^{p} d x \leq \eta^{p}
$$

provided

$$
\frac{1}{\left|B_{4}\right|} \int_{B_{4}^{+}}|\mathbf{f}|^{p} d x \leq \delta^{p}, \frac{1}{\left|B_{4}\right|} \int_{B_{4}^{+}}\left|A-\bar{A}_{B_{4}^{+}}\right|^{\frac{p}{p-1}} d x \leq \delta^{\frac{p}{p-1}} .
$$

Now choose any standard cut-off function $\phi \in C^{\infty}$ satisfying

$$
0 \leq \phi \leq 1, \operatorname{spt} \phi \subset B_{3} \text {, and } \phi=1 \text { on } \overline{B_{2}} .
$$


Then without loss of generality we assume $\phi^{p}(u-v) \in C_{0}^{\infty}\left(B_{4}^{+}\right)$by approximation. Now according to Definition 4.1 we have

$$
\int_{B_{4}^{+}}(A \nabla u \cdot \nabla u)^{\frac{p-2}{2}} A \nabla u \cdot \nabla\left(\phi^{p}(u-v)\right) d x=\int_{B_{4}^{+}}|\mathbf{f}|^{p-2} \mathbf{f} \cdot \nabla\left(\phi^{p}(u-v)\right) d x
$$

and

$$
\int_{B_{4}^{+}}\left(\bar{A}_{B_{4}^{+}} \nabla v \cdot \nabla v\right)^{\frac{p-2}{2}} \bar{A}_{B_{4}^{+}} \nabla v \cdot \nabla\left(\phi^{p}(u-v)\right) d x=0 .
$$

Subtracting the identity (4.25) from the identity (4.24) and operating basic computations we write the resulting expression as

$$
I_{1}=I_{2}+I_{3}+I_{4}+I_{5}+I_{6}
$$

for

$$
\begin{aligned}
& I_{1}=\int_{B_{4}^{+}} \phi^{p}\left((A \nabla u \cdot \nabla u)^{\frac{p-2}{2}} A \nabla u-(A \nabla v \cdot \nabla v)^{\frac{p-2}{2}} A \nabla v\right) \cdot(\nabla u-\nabla v) d x, \\
& I_{2}=-p \int_{B_{4}^{+}} \phi^{p-1}(u-v)(A \nabla u \cdot \nabla u)^{\frac{p-2}{2}} A \nabla u \cdot \nabla \phi d x, \\
& I_{3}=p \int_{B_{4}^{+}} \phi^{p-1}(u-v)(A \nabla v \cdot \nabla v)^{\frac{p-2}{2}} A \nabla v \cdot \nabla \phi d x, \\
& I_{4}=\int_{B_{4}^{+}}\left(\phi^{p-1}(u-v)|\mathbf{f}|^{p-2} \mathbf{f} \cdot \nabla \phi+\phi^{p}|\mathbf{f}|^{p-2} \mathbf{f} \cdot \nabla(u-v)\right) d x, \\
& I_{5}=\int_{B_{4}^{+}}\left((A \nabla v \cdot \nabla v)^{\frac{p-2}{2}} A \nabla v-\left(\bar{A}_{B_{4}^{+}} \nabla v \cdot \nabla v\right)^{\frac{p-2}{2}} \bar{A}_{B_{4}^{+}} \nabla v\right) \cdot \phi^{p} \nabla(u-v) d x, \\
& I_{6}=p \int_{B_{4}^{+}}\left((A \nabla v \cdot \nabla v)^{\frac{p-2}{2}} A \nabla v-\left(\bar{A}_{B_{4}^{+}} \nabla v \cdot \nabla v\right)^{\frac{p-2}{2}} \bar{A}_{B_{4}^{+}} \nabla v\right) \cdot \phi^{p-1}(u-v) \nabla \phi .
\end{aligned}
$$

Estimate of $I_{1}$. We divide it into two cases.

Case 1. $p \geq 2$. Using the elementary inequality

$$
\left((A \xi \cdot \xi)^{\frac{p-2}{2}} A \xi-(A \eta \cdot \eta)^{\frac{p-2}{2}} A \eta\right) \cdot(\xi-\eta) \geq C|\xi-\eta|^{p}
$$

for every $\xi, \eta \in \mathbb{R}^{n}$, we have

$$
I_{1} \geq C \int_{B_{4}^{+}}|\phi \nabla(u-v)|^{p} d x
$$

Case 2. $1<p<2$. Using the elementary inequality

$$
|\xi-\eta|^{p} \leq C(p) \tau^{\frac{p-2}{p}}\left(|\xi|^{p-2} \xi-|\eta|^{p-2} \eta\right) \cdot(\xi-\eta)+\tau|\eta|^{p}
$$

for every $\xi, \eta \in \mathbb{R}^{n}$ and for every $\tau \in(0,1]$, we have

$$
I_{1}+\tau \int_{B_{4}^{+}} \phi^{p}|\nabla v|^{p} d x \geq C(\tau) \int_{B_{4}^{+}}|\phi \nabla(u-v)|^{p} d x .
$$

Estimate of $I_{2}$. Since $A \in L^{\infty}$, we readily check from the uniform ellipticity condition and Young's inequality with $\tau$ that

$$
\begin{aligned}
I_{2} & \leq C \int_{B_{4}^{+}}(|\phi||\nabla u|)^{p-1}(|u-v \| \nabla \phi|) d x \\
& \leq \tau \int_{\Omega_{4}}|\phi|^{p}|\nabla u|^{p} d x+C(\tau) \int_{B_{4}^{+}}|u-v|^{p}|\nabla \phi|^{p} d x .
\end{aligned}
$$


Estimate of $I_{3}$. Similarly to the estimate of $I_{2}$, we have

$$
I_{3} \leq \tau \int_{B_{4}^{+}}|\phi|^{p}|\nabla v|^{p} d x+C(\tau) \int_{B_{4}^{+}}|u-v|^{p}|\nabla \phi|^{p} d x .
$$

Estimate of $I_{4}$. From Young's inequality with $\tau$ we observe

$$
\begin{aligned}
I_{4} \leq & C \int_{B_{4}^{+}}(|\phi||\mathbf{f}|)^{p-1}(|u-v||\nabla \phi|)+(|\phi||\mathbf{f}|)^{p-1}(|\phi||\nabla(u-v)|) d x \\
\leq & \tau \int_{B_{4}^{+}}|\nabla \phi|^{p}|u-v|^{p} d x \\
& +C(\tau) \int_{B_{4}^{+}}|\phi|^{p}|\mathbf{f}|^{p} d x+\tau \int_{B_{4}^{+}}|\phi \nabla(u-v)|^{p} d x .
\end{aligned}
$$

Estimate of $I_{5}$. Using the elementary inequality

$$
\left|(A \xi \cdot \xi)^{\frac{p-2}{2}} A \xi-(\widetilde{A} \xi \cdot \xi)^{\frac{p-2}{2}} \widetilde{A} \xi\right| \leq C(p, \Lambda)|A-\widetilde{A}||\xi|^{p-1}
$$

for every $\xi, \eta \in \mathbb{R}^{n}$ and from Young's inequality with $\tau$, we have

$$
\begin{aligned}
I_{5} & \leq C \int_{B_{4}^{+}}\left(|A-\widetilde{A}|(|\phi||\nabla v|)^{p-1}\right)|\phi \nabla(u-v)| d x \\
& \leq \tau \int_{B_{4}^{+}}|\phi \nabla(u-v)|^{p} d x+C(\tau) \int_{B_{4}^{+}}\left|A-\bar{A}_{B_{4}}\right|^{\frac{p}{p-1}}|\phi \nabla v|^{p} d x \\
& \leq \tau \int_{B_{4}^{+}}|\phi \nabla(u-v)|^{p} d x+C(\tau) \int_{B_{4}^{+}}\left|A-\bar{A}_{B_{4}}\right|^{\frac{p}{p-1}} d x,
\end{aligned}
$$

the last inequality following from the interior $W^{1, \infty}$ regularity for $v$ and (4.23).

Estimate of $I_{6}$. Similarly to the estimate of $I_{5}$, we have

$$
\begin{aligned}
I_{6} & \leq C \int_{B_{4}^{+}}\left(\left|A-\bar{A}_{B_{4}}\right|(|\phi||\nabla v|)^{p-1}\right)|(u-v) \nabla \phi| d x \\
& \leq \tau \int_{\Omega_{4}}|u-v|^{p}|\nabla \phi|^{p} d x+C(\tau) \int_{B_{4}^{+}}\left|A-\bar{A}_{B_{4}}\right|^{\frac{p}{p-1}}|\phi \nabla v|^{p} d x \\
& \leq \tau \int_{B_{4}^{+}}|\nabla \phi|^{p}|(u-v)|^{p} d x+C(\tau) \int_{B_{4}^{+}}\left|A-\bar{A}_{B_{4}}\right|^{\frac{p}{p-1}} d x .
\end{aligned}
$$

Using (4.23) and combining all the estimates $I_{1}$ to $I_{6}$, we have

$$
\begin{aligned}
& C(\Lambda, \tau) \int_{B_{4}^{+}}|\phi \nabla(u-v)|^{p} d x \\
& \leq C \cdot \tau \int_{B_{4}^{+}}|\phi \nabla(u-v)|^{p} d x \\
& +\tau \int_{B_{4}^{+}}\left(|\nabla u|^{p}+|\nabla v|^{p}\right) d x+C(\tau) \int_{B_{4}^{+}}|u-v|^{p} d x \\
& +C(\tau) \int_{B_{4}^{+}}|\mathbf{f}|^{p} d x+C(\tau) \int_{B_{4}^{+}}\left|A-\bar{A}_{B_{4}}\right|^{\frac{p}{p-1}} d x .
\end{aligned}
$$

Using (4.23) and selecting a sufficiently small constant $\tau>0$, we find

$$
\int_{B_{2}^{+}}|\nabla(u-v)|^{p} d x \leq C\left(\int_{B_{4}^{+}}|\nabla(u-v)|^{p} d x+\int_{B_{4}^{+}}|\mathbf{f}|^{p} d x+\int_{B_{4}^{+}}\left|A-\bar{A}_{B_{4}}\right|^{\frac{p}{p-1}} d x\right) \text {. }
$$


Then (4.21)-(4.22) imply

$$
\int_{B_{2}^{+}}|\nabla(u-v)|^{p} d x \leq C\left(\eta^{p}+\delta^{p}+\delta^{\frac{p}{p-1}}\right),
$$

where $\eta=\eta(\delta)$ is to be selected later.

According to $W^{1, \infty}$ interior regularity for $v$ there exists a constant $N_{0}$ such that

$$
\|\nabla v\|_{L^{\infty}\left(B_{3}^{+}\right)}^{p} \leq N_{0}^{p} .
$$

Denote by $N_{1}$ by the constant $N_{1}^{p}=\max \left\{2^{p+1} N_{0}^{p}, 2^{n}\right\}$. We claim

$$
\left\{x \in B_{1}^{+}: \mathcal{M}\left(|\nabla u|^{p}>N_{1}^{p}\right)\right\} \subset\left\{x \in B_{1}^{+}: \mathcal{M}_{B_{4}^{+}}\left(\mid \nabla\left(u-\left.v\right|^{p}\right)>N_{0}^{p}\right\} .\right.
$$

To see this, now suppose that

$$
x_{1} \in\left\{x \in B_{1}^{+}: \mathcal{M}_{B_{4}^{+}}\left(|\nabla(u-v)|^{p}\right)(x) \leq N_{0}^{p}\right\} .
$$

If $r \leq 2, B_{r}^{+}\left(x_{1}\right) \subset B_{3}^{+}$. Thus we observe from (4.29), (4.27) that

$$
\frac{1}{\left|B_{r}\right|} \int_{B_{r}^{+}\left(x_{1}\right)}|\nabla u|^{p} d x \leq \frac{2^{p}}{\left|B_{r}\right|} \int_{B_{r}^{+}\left(x_{1}\right)}\left(|\nabla(u-v)|^{p}+|\nabla v|^{p}\right) d x \leq 2^{p+1} N_{0}^{p}
$$

If $r>2, B_{r}^{+}\left(x_{1}\right) \subset B_{2 r}^{+}\left(x_{0}\right)$, and so (4.18) implies

$$
\frac{1}{\left|B_{r}\right|} \int_{B_{r}^{+}\left(x_{1}\right)}|\nabla u|^{p} d x \leq \frac{2^{n}}{\left|B_{2 r}\right|} \int_{B_{2 r}^{+}\left(x_{0}\right)}|\nabla u|^{p} d x \leq 2^{n} .
$$

Using (4.30) and (4.31) we conclude that

$$
x_{1} \in\left\{x \in B_{1}^{+}: \mathcal{M}\left(|\nabla u|^{p}\right)(x) \leq N_{1}^{p}\right\} .
$$

Assertion (4.28) comes from (4.30) and (4.31). We consequently can calculate from (4.28), a weak $(1,1)$ estimate (see Lemma 2.5$)$ and (4.26)

$$
\begin{aligned}
\left|\left\{x \in B_{1}^{+}: \mathcal{M}\left(|\nabla u|^{p}\right)>N_{1}^{p}\right\}\right| & \leq\left|\left\{x \in B_{1}^{+}: \mathcal{M}_{B_{4}^{+}}\left(|\nabla(u-v)|^{p}\right)>N_{0}^{p}\right\}\right| \\
& \leq \frac{C}{N_{0}^{p}} \int_{B_{2}^{+}}|\nabla(u-v)|^{p} d x \\
& \leq \frac{1}{N_{0}^{p}} C\left(\eta^{p}+\delta^{p}+\delta^{\frac{p}{p-1}}\right) .
\end{aligned}
$$

Consequently we have

$$
\left|\left\{x \in B_{1}^{+}: \mathcal{M}\left(|\nabla u|^{p}\right)>N_{1}^{p}\right\}\right| \leq C\left(\eta^{p}+\delta^{p}+\delta^{\frac{p}{p-1}}\right)=\epsilon\left|B_{1}\right|,
$$

provided that we select $\eta=\eta(\delta), \delta$ satisfying the last identity above. This completes the proof.

We now come to state the scaling invariant form of the lemma above. We have

Corollary 4.4. There exists a constant $N_{1}>0$ so that for any $0<\epsilon, r<1$, there exists a small $\delta=\delta(\epsilon)>0$ such that if $u$ is a weak solution of (4.1) in $B_{6}^{+}$, with $A$ 
$(\delta, 6 r)$-vanishing and

$$
B_{r}^{+} \cap\left\{x: \mathcal{M}\left(|\nabla u|^{p}\right)(x) \leq 1\right\} \cap\left\{x: \mathcal{M}\left(|\mathbf{f}|^{p}\right)(x) \leq \delta^{p}\right\} \neq \emptyset,
$$

then we have

$$
\left|\left\{x \in B_{6}^{+}: \mathcal{M}\left(|\nabla u|^{p}\right)(x)>N_{1}^{p}\right\} \cap B_{r}\right|<\epsilon\left|B_{r}\right| .
$$

Lemma 4.5. There is a constant $N_{1}>0$ so that for any $0<\epsilon, r \leq 1$, there exists a small $\delta=\delta(\epsilon)>0$ such that if $u$ is a weak solution of (4.1) in $B_{42}^{+}(0)$, with $(\delta, 42)$-vanishing and

$$
\left|\left\{x \in B_{1}^{+}(0): \mathcal{M}\left(|\nabla u|^{p}\right)(x)>N_{1}^{p}\right\} \cap B_{r}\right| \geq \epsilon\left|B_{r}\right|,
$$

then we have

$$
B_{r} \cap B_{1}^{+}(0) \subset\left\{x \in B_{1}^{+}(0): \mathcal{M}\left(|\nabla u|^{p}\right)>1\right\} \cup\left\{x \in B_{1}^{+}(0): \mathcal{M}\left(|\mathbf{f}|^{p}\right)>\delta^{p}\right\},
$$

where $B_{r}$ denotes the ball with radius $r$ and center in $B_{1}^{+}(0)$.

Proof. We argue by contradiction. If $B_{r}$ satisfies (4.33) and the conclusion (4.34) is false, then there exists $x_{0} \in B_{r} \cap B_{1}^{+}(0)$ such that

$$
\frac{1}{\left|B_{\rho}\right|} \int_{B_{\rho}\left(x_{0}\right)}|\nabla u|^{p} d x \leq 1, \frac{1}{\left|B_{\rho}\right|} \int_{B_{\rho}\left(x_{0}\right)}|\mathbf{f}|^{p} d x \leq \delta^{p}
$$

for all $\rho>0$. If $B_{6 r} \cap\left\{x_{n}=0\right\}=\emptyset$, this is an interior estimate (see Lemma 3.3). So suppose that $\left(x^{\prime}, 0\right) \in B_{6 r} \cap\left\{x_{n}=0\right\}$. Now observe

$$
B_{6 r}^{+} \subset B_{7 r}^{+}\left(x^{\prime}, 0\right) .
$$

Applying Corollary 4.4 to the ball $B_{7 r}^{+}\left(x^{\prime}, 0\right)$, with $\frac{\epsilon}{7^{n}}$ replacing $\epsilon$ we obtain

$$
\begin{aligned}
& \left|\left\{x \in B_{1}^{+}: \mathcal{M}\left(|\nabla u|^{p}\right)(x)>N_{1}^{p}\right\} \cap B_{r}\right| \\
& \leq\left|\left\{x \in B_{1}^{+}: \mathcal{M}\left(|\nabla u|^{p}\right)(x)>N_{1}^{p}\right\} \cap B_{7 r}^{+}\left(x^{\prime}, 0\right)\right| \\
& <\frac{\epsilon}{7^{n}}\left|B_{7 r}^{+}\right| \\
& =\epsilon\left|B_{r}^{+}\right| .
\end{aligned}
$$

Then we reach a contradiction to (4.33).

Now take $N_{1}, \epsilon$, and the corresponding $\delta>0$ given by the lemma above and set

$$
\epsilon_{1}=2(10)^{n} \epsilon
$$

Corollary 4.6. Let $u$ be a weak solution of (4.1) in $B_{42}^{+}$and $k$ be a positive integer. Assume that $A$ is $(\delta, 42)$-vanishing. Assume further that

$$
\left|\left\{x \in B_{1}^{+}: \mathcal{M}\left(|\nabla u|^{p}\right)>N_{1}^{p}\right\}\right|<\epsilon\left|B_{1}\right| .
$$

Then we have

$$
\begin{aligned}
& \left|\left\{x \in B_{1}^{+}: \mathcal{M}\left(|\nabla u|^{p}\right)>\left(N_{1}^{p}\right)^{k}\right\}\right| \\
& \leq \sum_{i=1}^{k} \epsilon_{1}^{i}\left|\left\{x \in B_{1}^{+}: \mathcal{M}\left(|\mathbf{f}|^{p}\right)>\delta^{p}\left(N_{1}^{p}\right)^{k-i}\right\}\right|+\epsilon_{1}^{k}\left|\left\{x \in B_{1}^{+}: \mathcal{M}\left(|\nabla u|^{p}\right)>1\right\}\right| .
\end{aligned}
$$


Proof. We want to prove this lemma by induction on $k$. The case $k=1$ follows from Lemma 4.5 and Lemma 2.6 on

$$
\begin{aligned}
C & =\left\{x \in B_{1}^{+}: \mathcal{M}\left(|\nabla u|^{p}\right)>N_{1}^{p}\right\}, \\
D & =\left\{x \in B_{1}^{+}: \mathcal{M}\left(|\mathbf{f}|^{p}\right)>\delta^{p}\right\} \cup\left\{x \in B_{1}^{+}: \mathcal{M}\left(|\nabla u|^{p}\right)>1\right\} .
\end{aligned}
$$

Suppose then that the conclusion is valid for some positive integer $k \geq 2$. Set $u_{1}=\frac{u}{N_{1}}$ and $\mathbf{f}_{1}=\frac{\mathbf{f}}{N_{1}}$. Thus $u_{1}$ is the weak solution of

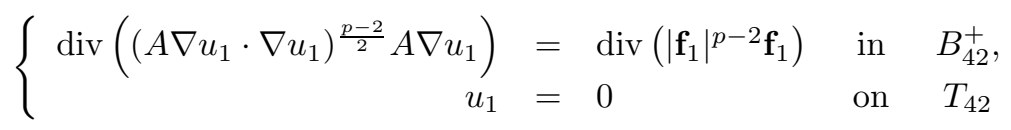

and

$$
\left|\left\{x \in B_{1}^{+}: \mathcal{M}\left(\left|\nabla u_{1}\right|^{p}\right)(x)>N_{1}^{p}\right\}\right|<\epsilon\left|B_{1}\right| .
$$

Then by the induction assumption

$$
\begin{aligned}
& \left|\left\{x \in B_{1}^{+}: \mathcal{M}\left(|\nabla u|^{p}\right)>N_{1}^{(k+1) p}\right\}\right| \\
& =\left|\left\{x \in B_{1}^{+}: \mathcal{M}\left(\left|\nabla u_{1}\right|^{p}\right)>N_{1}^{k p}\right\}\right| \\
& \leq \sum_{i=1}^{k} \epsilon_{1}^{i}\left|\left\{x \in B_{1}^{+}: \mathcal{M}\left(\left|\mathbf{f}_{1}\right|^{p}\right)>\delta^{p} N_{1}^{(k-i) p}\right\}\right| \\
& +\epsilon_{1}^{k}\left|\left\{x \in B_{1}^{+}: \mathcal{M}\left(\left|\nabla u_{1}\right|^{p}\right)>1\right\}\right| \\
& =\sum_{i=1}^{k+1} \epsilon_{1}^{i}\left|\left\{x \in B_{1}^{+}: \mathcal{M}\left(|\mathbf{f}|^{p}\right)>\delta^{p} N_{1}^{(k+1-i) p}\right\}\right| \\
& +\epsilon_{1}^{k+1}\left|\left\{x \in B_{1}^{+}: \mathcal{M}\left(|\nabla u|^{p}\right)>1\right\}\right| .
\end{aligned}
$$

These estimates in turn complete the induction on $k$.

Finally, in view of Corollary 4.6 we have the following boundary estimates.

Theorem 4.7. Let $q$ be a real number with $q>p$. There is a small $\delta=\delta(p, n, \Lambda)>$ 0 so that if $u$ is a weak solution of (4.1) in $B_{42}^{+}$, with $A$ uniformly elliptic and $(\delta, 42)$ vanishing and $\mathbf{f} \in L^{q}\left(B_{42}^{+}, \mathbb{R}^{n}\right)$, then $u$ belongs to $W^{1, q}\left(B_{1}^{+}\right)$with the estimate

$$
\int_{B_{1}^{+}}|\nabla u|^{q} d x \leq C \int_{B_{6}^{+}}\left(|u|^{q}+|\mathbf{f}|^{q}\right) d x,
$$

where the constant $C$ is independent of $u$ and $\mathbf{f}$.

Proof. According to standard arguments of measure theory (See Lemma 2.7), there exists a constant $C=C\left(\delta, N_{1}^{p}, q\right)$ such that

$$
\sum_{k=1}^{\infty}\left(N_{1}^{p}\right)^{k \frac{q}{p}}\left|\left\{x \in B_{6}^{+}: \mathcal{M}\left(|\mathbf{f}|^{p}\right)>\delta^{p}\left(N_{1}^{p}\right)^{k}\right\}\right| \leq C\left\|\mathcal{M}\left(|\mathbf{f}|^{p}\right)_{L^{\frac{q}{p}\left(B_{6}^{+}\right)}}^{\frac{q}{p}} \leq C\right\| \mathbf{f} \|_{L^{q}\left(B_{6}^{+}\right)}^{q} .
$$

The last estimate follows from the $L^{q}$-estimate of the Hardy-Littlewood maximal function. Now we may with no loss suppose

$$
\left|\left\{x \in \Omega: \mathcal{M}\left(|\nabla u|^{p}\right)>N_{1}^{p}\right\} \cap B_{1}^{+}\right|<\epsilon\left|B_{1}\right| .
$$


Then from Corollary 4.6 and (4.36) we have

$$
\begin{aligned}
& \sum_{k=1}^{\infty}\left(N_{1}^{p}\right)^{k \frac{q}{p}}\left|\left\{x \in B_{1}^{+}: \mathcal{M}\left(|\nabla u|^{p}\right)>\left(N_{1}^{p}\right)^{k}\right\}\right| \\
& \leq \sum_{k=1}^{\infty} N_{1}^{q k}\left(\sum_{i=1}^{k} \epsilon_{1}^{i}\left|\left\{x \in B_{1}^{+}: \mathcal{M}\left(|\mathbf{f}|^{p}\right)>\delta^{p} N_{1}^{p(k-i)}\right\}\right|\right. \\
& \left.+\epsilon_{1}^{k}\left|\left\{x \in B_{1}^{+}: \mathcal{M}\left(|\nabla u|^{p}\right)>1\right\}\right|\right) \\
& =\sum_{i=1}^{\infty}\left(N_{1}^{q} \epsilon_{1}\right)^{i}\left(\sum_{k=i}^{\infty}\left(N_{1}^{p}\right)^{(k-i) \frac{q}{p}}\left|\left\{x \in B_{1}^{+}: \mathcal{M}\left(|\mathbf{f}|^{p}\right)>\delta^{p} N_{1}^{p(k-i)}\right\}\right|\right) \\
& +\sum_{k=1}^{\infty}\left(N_{1}^{q} \epsilon_{1}\right)^{k}\left|\left\{x \in B_{6}^{+}: \mathcal{M}\left(|\nabla u|^{p}\right)>1\right\}\right| \\
& \leq C\|\mathbf{f}\|_{L^{q}\left(B_{6}^{+}\right)}^{q} \sum_{k=1}^{\infty}\left(N_{1}^{q} \epsilon_{1}\right)^{k} \\
& \leq C\|\mathbf{f}\|_{L^{q}\left(B_{6}^{+}\right)}^{q}
\end{aligned}
$$

provided $\epsilon>0$ is selected small enough to have

$$
\left(N_{1}^{q} \epsilon_{1}\right)=\left(N_{1}^{q}\right) 2(10)^{n} \epsilon<1 \text {. }
$$

Then

$$
\sum_{k=1}^{\infty}\left(N_{1}^{p}\right)^{k \frac{q}{p}}\left|\left\{x \in B_{1}^{+}: \mathcal{M}\left(|\nabla u|^{p}\right)>\left(N_{1}^{p}\right)^{k}\right\}\right| \leq C\|\mathbf{f}\|_{L^{q}\left(B_{6}^{+}\right)}^{q} .
$$

Thus we have from Lemma 2.5 that

$$
\mathcal{M}\left(|\nabla u|^{p}\right) \in L^{\frac{q}{p}}\left(B_{1}^{+}\right)
$$

and

with the estimate

$$
\nabla u \in L^{q}\left(B_{1}^{+} ; \mathbb{R}^{n}\right)
$$

$$
\|\nabla u\|_{L^{q}\left(B_{1}^{+}\right)} \leq\left(\|u\|_{L^{q}\left(B_{6}^{+}\right)}+\|\mathbf{f}\|_{L^{q}\left(B_{6}^{+}\right)}\right)
$$

and we are done.

\section{Flattening ARgument}

In the general case we choose any point $x_{0} \in \partial \Omega$. We may assume that

$$
\Omega \cap B_{r}\left(x_{0}\right)=\left\{x \in B_{r}\left(x_{0}\right): x_{n}>\gamma\left(x^{\prime}\right)\right\}
$$

for some constant $r>0$ and some Lipschitz continuous function

$$
\gamma: \mathbb{R}^{n-1} \rightarrow \mathbb{R}
$$

with $\operatorname{Lip}(\gamma)$ small, where $\operatorname{Lip}(\gamma)$ denotes the Lipschitz constant of $\gamma$.

Now define

$$
y_{i}=x_{i}=\Phi^{i}(x)(1 \leq i \leq n-1) \text { and } y_{n}=x_{n}-\gamma\left(x^{\prime}\right)=\Phi^{n}(x)
$$

and write

$$
y=\Phi(x) \text { and } x=\Phi^{-1}(y)=\Psi(y) .
$$


Choose $s>0$ so small that $B_{s}^{+}$lies in $\Phi\left(\Omega \cap B_{r}\left(x_{0}\right)\right)$ and define

$$
u_{1}(y)=u(\Psi(y))
$$

for all $y \in B_{s}^{+}$.

If $u$ is a weak solution of

$$
\left\{\begin{aligned}
\operatorname{div}\left((A \nabla u \cdot \nabla u)^{\frac{p-2}{2}} A \nabla u\right) & =\operatorname{div}\left(|\mathbf{f}|^{p-2} \mathbf{f}\right) & & \text { in } & \Omega \cap B_{r}\left(x_{0}\right), \\
u & =0 & & \text { on } & \partial \Omega \cap B_{r}\left(x_{0}\right),
\end{aligned}\right.
$$

then $u_{1}$ is a weak solution of

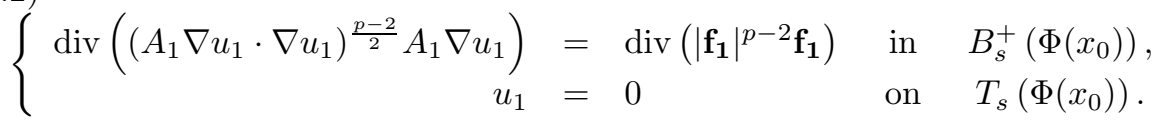

Here

$$
A_{1}(y)=[\nabla \Phi]^{T}(\Psi(y) \cdot A(\Psi(y)) \cdot[\nabla \Phi](\Psi(y))
$$

and

$$
\mathbf{f}_{1}(y)=[\nabla \Phi]^{T}(\Psi(y)) \cdot \mathbf{f}(\Psi(y)) .
$$

Then it is straightforward to check that

$$
\left[A_{1}\right]_{B M O} \leq C\left([A]_{B M O}+\|\nabla \gamma\|_{L^{\infty}\left(\mathbb{R}^{n-1}\right)}\right) \leq C\left([A]_{B M O}+\operatorname{Lip}(\gamma)\right),
$$

where $\left[A_{1}\right]_{B M O}$ denotes the BMO seminorm of $A_{1}$ and $[A]_{B M O}$ denotes the BMO seminorm of $A$.

Now we remark that $\gamma$ in the above is Lipschitz continuous with small Lipschitz constant if and only if it is in $W^{1, \infty}$ with small $\|\nabla \gamma\|_{L^{\infty}}$ (see Theorem 4 of chapter 5 in [5]). Recalling the assumptions announced in the Introduction that $\Omega$ is $(\delta, R)$ Lipschitz and that $A$ is $(\delta, R)$-vanishing, it follows easily from (5.5) and (5.3) that $A_{1}$ is $(\delta, R)$-vanishing and that $A_{1}$ is uniformly elliptic. Hence we can find a boundary estimate for the case that $\Omega$ is $(\delta, R)$-Lipschitz and $A$ is $(\delta, R)$-vanishing via the approached used in Section 4.

We are finally set to give our proof of Theorem 1.2.

Proof. Once we established the boundary $L^{q}(q>p)$ estimates for the gradient of $u$ in $B_{1}^{+}$in Theorem 4.7 we can get the proof by standard scaling, covering and flattening arguments along with the interior estimate and a duality argument.

\section{ACKNOWLEDGMENTS}

The authors wish to thank Professor Shulin Zhou for some very helpful conversations on this work.

\section{REFERENCES}

1. S. Byun, Elliptic equations with BMO coefficients in Lipschitz domains, Trans. Amer. Math. Soc., 357 (2005), 1025-1046. MR2110431 (2005i:35054)

2. L.A. Caffarelli and X. Cabré, Fully nonlinear elliptic equations, vol. 43, Amer. Math. Soc., Providence, RI, 1995. MR1351007 (96h:35046)

3. L.A. Caffarelli and I. Peral, On $W^{1, p}$ estimates for elliptic equations in divergence form, Comm. Pure Appl. Math., 51, (1998), 1-21. MR1486629 (99c:35053) 
4. E. DiBenedetto and J. Manfredi, On the higher integrability of the gradient of weak solutions of certain degenerate elliptic systems, Amer. J. Math., 115 (5), 1993, 1107-1134. MR1246185 (94i:35077)

5. L.C. Evans, Partial Differential Equations, Graduate Studies in Mathematics, 19, American Mathematical Society, Providence, RI, (1998), xviii+662 pp. ISBN: 0-8218-0772-2. MR1625845 (99e:35001)

6. T. Iwaniec, Projections onto gradient fields and $L^{p}$-estimates for degenerate elliptic operators, Studia Math., 75, 1983, 293-312. MR0722254 (85i:46037)

7. D. Jerison and C. Kenig, The inhomogeneous Dirichlet problem in Lipschitz domains, J. Funct. Anal., 130, (1995), 161-219. MR1331981 (96b:35042)

8. D. Jerison and C. Kenig, The logarithm of the Poisson kernel of a $C^{1}$ domain has vanishing mean oscillation, Trans. Amer. Math. Soc. 273, (1982), 781-794. MR0667174 (83k:31004)

9. D. Jerison and C. Kenig, The Neumann problem on Lipschitz domains, Bull. Amer. Math. Soc. (N.S.), 4, (1981), 203-207. MR0598688 (84a:35064)

10. J. Kinnunen and S. Zhou, A Local estimate for nonlinear equations with discontinuous coefficients, Comm. partial differential equations, 24, (1999), 2043-2068. MR1720770 (2000k:35084)

11. J. Kinnunen and S. Zhou, A boundary estimate for nonlinear equations with discontinuous coefficients, Differential and integral equations, 14 (2001), 475-492. MR1799417 (2002a:35070)

12. P.W. Jones, Extension theorems for BMO, Indiana Univ. Math. J., 29, (1980), 41-66. MR0554817 (81b:42047)

13. E. M. Stein, Harmonic Analysis, Princeton University Press, Princeton, NJ, 1993. MR1232192 (95c:42002)

14. L. Wang, A geometric approach to the Calderón-Zygmund estimates, Acta Mathematica Sinica, 19, (2003), 381-396. MR1987802 (2004e:42033)

Department of Mathematical Sciences, Seoul National University, Seoul 151-747, KOREA

E-mail address: byun@math.snu.ac.kr

Department of Mathematics, University of Iowa, Iowa City, Iowa 52242 - And - College of Sciences, Xian Jiaotong University, Xian 710049, People's Republic of China

E-mail address: lwang@math.uiowa.edu 